\section{El UlTRASONIDO Y SU ApLICACIÓN}

(1) Eulogio Santos De La Cruz

(2) Néstor Cancino Vera

(3) Julio Yenque Dedios

(4) David Ramírez Morales

(5) Máximo Palomino Pérez

\section{RESUMEN}

El ultrasonido es utilizado para el ensayo no destructivo de los materiales, se aplica para conocer el interior de un material o componentes a procesar la trayectoria de la propagación de las ondas sonoras, se conoce que la propagación de las ondas en el interior de la pieza dependen de las discontinuidades del material examinado, lo que permite evaluar aquella discontinuidad acerca de su forma, tamaño, orientación, ya que estos oponen resistencia conocida como impedancia acústica. Al conocer la propagación de las ondas sonoras, se determina su velocidad con ello las propiedades de los materiales.

Palabras Clave: Ultrasonido. Velocidad. Onda.

UITRASOUND AND ITS APPLICATION ABSTRACT

Ultrasound is used for the non-destructive testing of materials. It is applied to know the inner of a material or components when processing the trajectory of the propagation of sound waves. It is know that propagation of waves inside the piece depends on the discotinuities of the material examined, which allows to evaluate the discontinuity about its form, size, direction, since these offer a resistance known as acoustic impedance. When knowing the propagation of the sound waves, its speed and therefore the property of materials are determined.

Key words: Ultrasound. Speed. Wave.

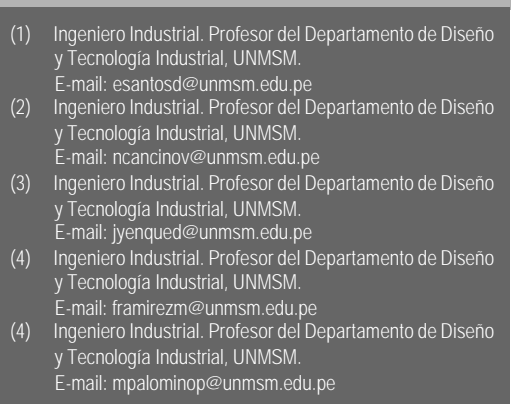

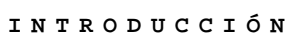

El método del ultrasonido en la ingeniería es utilizado para el ensayo de los materiales, es una técnica de ensayo no destructivo (END) y tiene diversas aplicaciones, en especial para conocer el interior de un material o sus componentes según la trayectoria de la propagación de las ondas sonoras, al procesar las señales de las ondas sonoras se conoce el comportamiento de las mismas durante su propagación en el interior de la pieza y que dependen de las discontinuidades del material examinado, lo que permite evaluar aquella discontinuidad acerca de su forma, tamaño, orientación, debido que la discontinuidad opone resistencia (conocida como impedancia acústica) al paso de una onda. Las ondas pueden ser sónicas comprendidas en el intervalo de frecuencias entre 20 y $500 \mathrm{kHz}$ y las ultrasónicos con frecuencias superiores a $500 \mathrm{kHz}$.

En el método ultrasónico se utilizan instrumentos que transmiten ondas con ciertos intervalos de frecuencia y se aplican para detectar defectos como poros, fisuras, también para conocer las propiedades básicas de los líquidos y sólidos como la composición, estructura.

El análisis de los materiales mediante ultrasonido se basa en el principio físico: El movimiento de una onda acústica, sabido es que la onda acústica es afectada por el medio a través del cual viaja y se distinguen los siguientes tipos: onda longitudinal, transversal y superficial (Rayleigh), según se muestra en la Figura 1, debido a ello ocurren los cambios asociados con el paso de una onda sonora de alta frecuencia a través de un material en uno o más de los cuatro parámetros siguientes: tiempo de tránsito, atenuación, reflexión y frecuencia. Estos parámetros a menudo pueden estar correlacionados con los cambios de las propiedades físicas, dureza, módulo de elasticidad, densidad, homogeneidad, estructura y grano del material.

MARCO

CONCEPTUAL

Nomenclatura

Se utiliza la siguiente nomenclatura:

V, velocidad del sonido en el material

$\mathbf{t}$, tiempo en segundos

$\lambda$, longitud de onda

$\mathbf{f}$, frecuencia

$\mathbf{R}$, coeficiente de reflexión (porcentaje de energía reflejada)

$\mathbf{Z}_{1}$, impedancia acústica en el primer material

$\mathbf{Z}_{2}$, impedancia acústica en el segundo material

s, espesor 


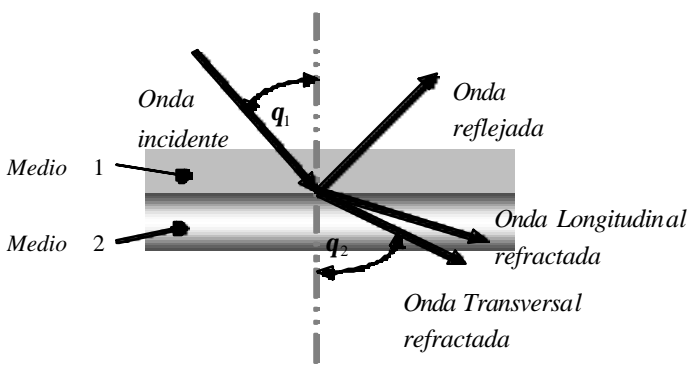

Figura 1. Transmisión de onda en dos medios diferentes

Principio

Las ondas sonoras son vibraciones mecánicas que viajan a través de un medio que puede ser un sólido, un líquido o, un gas, la propagación de las ondas a través del medio dado, es a una velocidad específica, dirección predecible y, cuando las ondas encuentran un límite con un medio distinto y con diferente impedancia mecánica, como se muestra en la Figura 2, las ondas se reflejarán o se transmitirán según reglas conocidas. Este es el principio físico utilizado para la detección de fallas en los materiales. La impedancia del medio conductor de ondas ultrasónicas se define mediante la siguiente expresión:

$$
Z=\rho v
$$

La impedancia de dos medios diferentes origina la reflexión, así como la transmisión y la amplitud de la onda, siendo útiles las siguientes relaciones:

$$
\begin{aligned}
& \text { Índice de reflexión: } I_{R}=\frac{\left(Z_{1}-Z_{2}\right)^{2}}{\left(Z_{1}+Z_{2}\right)^{2}} \\
& \text { Índice de transición: } I_{T}=\frac{4 Z_{1} Z_{2}}{\left(Z_{1}+Z_{2}\right)^{2}}
\end{aligned}
$$

Las amplitudes del haz incidente con el reflejado son: $\frac{A_{1}}{A_{2}}=\frac{Z_{1}-Z_{2}}{Z_{1}+Z_{2}}$

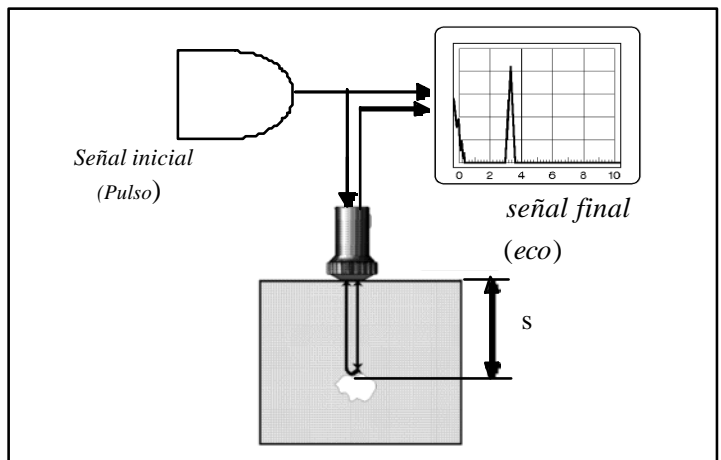

Figura 3. Esquema pulso-eco

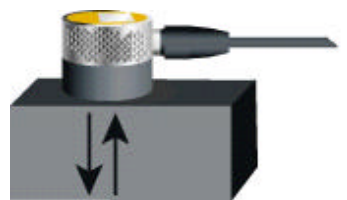

(a)

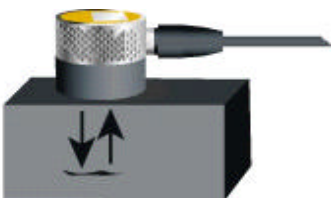

(b)
Figura 2. (a), la onda viaja libremente en el interior de la pieza hasta encontrarse con otro material para ser reflejado, la pieza no presenta defectos, en la figura 2 (b) la onda es reflejada por el defecto en el interior de la pieza

La naturaleza ultrasónica es debido a que tienen frecuencias altas y longitud de onda cortas, apropiadas para el ensayo de los materiales, para inspeccionar el interior de las piezas que ofrecen una trayectoria continua a la propagación de las ondas sonoras. Para generar onda ultrasónica, se utiliza un transductor piezoeléctrico que convierte las señales eléctricas en señales sonoras, y viceversa. El transductor consiste en un cristal (de cuarzo) piezoeléctrico insertado en un alojamiento a prueba de agua, que facilita su conexión eléctrica a un generador o transmisor-receptor de pulsos (modo pulso/ eco); en el modo de transmisión, se aplica al cristal un pulso de energía eléctrica de corta duración y alto voltaje, provocando que cambie rápidamente su configuración geométrica, deformándose, y emita un pulso de energía acústica (onda) de alta frecuencia. En el modo de recepción, cualquier onda ultrasónica o eco que regresen a través de la trayectoria acústica, la cual incluye los medios y partes de acoplamiento, comprimen el cristal, produciendo una señal eléctrica que se amplifica y se procesa en el receptor. Estos pulsos generados y recibidos por transductores piezoeléctricos deben estar acústicamente acoplados con el material que se ensaya, según el esquema mostrado en la Figura 3.

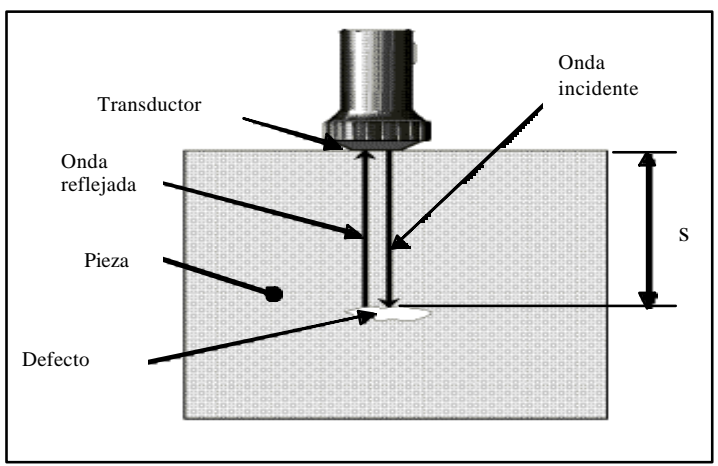

Figura 4. Reflexión de una onda debido a una discontinuidad 
La señal admitida es amplificada y analizada con una variedad de instrumentación comercial tanto analógica como digital disponible para este propósito, en la Figura 4 se muestra como una onda ultrasónica es reflejada al encontrarse con un defecto en el interior de la pieza.

Los parámetros a considerar son los siguientes:

\section{a. Velocidad}

La velocidad del sonido es usualmente el parámetro ultrasónico más fácil de medir, en un medio homogéneo está directamente relacionado con el módulo de elasticidad, densidad del material, módulo de Poisson, y el grado de homogeneidad. Según el tipo de onda la velocidad también es diferente. La velocidad se relaciona con la longitud y frecuencia de onda como sigue:

$$
\begin{gathered}
V=\lambda f \quad \text { o, } \quad V=\frac{\lambda}{T}, \\
\text { donde: } f=\frac{1}{T}
\end{gathered}
$$

Según la velocidad del sonido, la distancia recorrida por éste es: (ver Figura 4)

$$
s=\frac{V T}{2}
$$

Las velocidades de las ondas longitudinales y transversales también están influenciadas por las propiedades de los materiales, así por ejemplo en el acero SAE 1020 la velocidad de la onda longitudinal es $5,89 \times 10^{5} \mathrm{~cm} / \mathrm{s}$ y la transversal $3,24 \times 10^{5} \mathrm{~cm} / \mathrm{s}$; en el acero AISI 430 la longitudinal es $6,01 \times 10^{5} \mathrm{~cm} / \mathrm{s}$ y transversal $3,36 \times 10^{5} \mathrm{~cm} / \mathrm{s}$. De lo mencionado las constantes más importantes del material como módulo de elasticidad, cizallamiento y de Poisson están relacionadas con la velocidad de onda y son como sigue:

$$
\begin{gathered}
E=\frac{\left(3 V_{L}^{2}-4 V_{T}^{2}\right) V_{T}^{2} d}{V_{L}^{2}-V_{T}^{2}} \\
G=V_{T}^{2} d \\
\mu=\frac{V_{L}^{2}-2 V_{T}^{2}}{2\left(V_{L}^{2}-V_{T}^{2}\right)}
\end{gathered}
$$

\section{b. Atenuación}

Es la reducción del nivel de una señal, cuando pasa a través de un elemento; la intensidad de la energía aplicada disminuye con el espesor del material, siendo amortiguada en tasas diferentes según tipo de material, la amortiguación se debe a los efectos interactivos de la densidad, dureza, viscosidad, y estructura molecular. La atenuación en un material dado, normalmente aumenta con la frecuencia y se mide en decibeles, pero también se puede medir en porcentajes. El principal mecanismo de la atenuación de la onda es la absorción, que retiene y convierte en calor a la energía, la relación siguiente expresa la absorción:

$$
\begin{gathered}
E_{x}=E_{0} e^{-k x} \\
k=\frac{16 \pi^{2} f^{2} \eta}{3 V^{3} d}
\end{gathered}
$$

\section{c. Dispersión}

Las ondas acústicas rebotan según el material que se examina. Los cambios son disímiles dependiendo de la concentración, estructura, orientación de las fibras, porosidad, tamaño de la partícula, y otras variaciones microestructurales que afectan la amplitud, la dirección, y la frecuencia de las señales de onda. Los efectos de dispersión también pueden ser monitoreados indirectamente observando los cambios en la amplitud del eco o una señal de transmisión directa.

\section{d. Frecuencia (el Espectro)}

Todos los materiales tienden a actuar hasta cierto punto como un filtro al paso de la onda, atenuando o dispersándolo. Las ondas del sonido oscilan a una frecuencia específica, esto es, número de vibraciones o ciclos por segundo. El oído humano percibe hasta una frecuencia máxima de aproximadamente 20000 ciclos por segundo (20 KHz), mientras la mayoría de aplicaciones ultrasónicas utilizan frecuencias entre 500000 y 10 000000 ciclos por segundo ( $500 \mathrm{KHz}$ a $10 \mathrm{MHz})$. Las frecuencias altas son menos eficientes en el aire y otros gases, en la mayoría de los líquidos y materiales de ingeniería viaja libremente.

\section{e. Longitud de onda}

Cualquier tipo de onda tendrá una longitud asociada a la distancia entre dos puntos correspondiente de un ciclo de onda. La longitud de onda se relaciona con su frecuencia y su velocidad por una ecuación simple. Véase la Figura 5 con la ilustración de la longitud y periodo de una onda.

\section{A P I I C A C I O N E S}

Las aplicaciones son muy amplias y abarcan la industria metalúrgica, construcciones navales, aeronáuticas, y otras industrias en general; algunos ejemplos de aplicación son: 


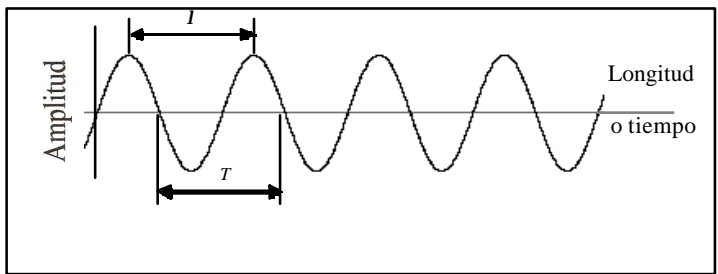

Figura 5. Longitud y frecuencia de onda

Módulo de elasticidad

El módulo de elasticidad (Young), módulo de cizallamiento, son calculados en función de la longitudinal y la velocidad de onda. Con el uso de técnicas especiales del método de ultrasonido las propiedades referidas de los materiales se pueden medir también a altas temperaturas.

Nodularidad de la fundición

Se cuantifica la concentración de grafito en hierro colado y su forma mediante la medición de la velocidad de la onda acústica.

Tasa de curado epoxi-concreto armado La razón de curado con epóxico y el hormigón se determina por cambio de velocidad de sonido cuando estos materiales se endurecen.

Concentración de líquidos

La proporción de una mezcla de dos líquidos a una temperatura dada puede estar correlacionada con la velocidad del sonido por ser disímiles según solución.

Proporción de mezclas

La proporción y la densidad de una mezcla líquido-sólido a una determinada temperatura se pueden correlacionar con la velocidad del sonido y/o atenuación.

Densidad de cerámicos

La densidad de la cerámica puede ser verificada midiendo la velocidad del sonido.

Productos alimenticios

La aplicación del ultrasonido por su condición de ser poco contaminante, se utiliza por ejemplo en la determinación de las edades de huevos y papas, madurez de frutas, contenido de grasa en carne roja, porcentaje de sólidos en la leche entre otros.

Polimerización en plásticos

En plásticos y otros polímeros, las variaciones en la estructura molecular como la longitud o la orientación de cadenas del polímero, a menudo están acom- pañadas de los cambios en la velocidad del sonido y/ o atenuación.

Dimensión y distribución de las partículas $y$ porosidades

Los cambios en el tamaño o la distribución de partículas o la porosidad en un medio sólido o líquido influyen en la amplitud y la frecuencia de ultrasonido.

Anisotropía en sólidos

Las variaciones de velocidad en el interior de las piezas, la dispersión, y/o la atenuación de ondas a través de diferentes ejes de un sólido pueden usarse para identificar y cuantificar la anisotropía de un material.

Profundidad de cementación en acero Las técnicas del ultrasonido especialmente de onda transversal y de alta frecuencia pueden usarse para medir la profundidad de cementación.

Medida de temperatura

La termometría ultrasónica se usa para medir temperaturas muy altas (por ejemplo a 3000 grados Celsius) monitoreando cambios en la velocidad del sonido.

C O N C L U S IONES

El principio físico del movimiento de una onda acústica, ha encontrado aplicaciones muy importantes tanto en la medicina como en la industria, particularmente el método del ultrasonido y su naturaleza no destructiva, en el ensayo de los materiales, para detectar los defectos interiores, deducir sus propiedades, así como conocer la corrosión y su magnitud, aparición de fisuras en partes de equipos, maquinarias e instalaciones con ello predecir la necesidad de sustituir piezas de las máquinas, reparación, entre otros.

B I B I I O G R A F Í A

1. Farnell, G.W. y L. Adler. (1972). Elastic wave propagation in thin layers. En: Physical Acoustics, vol. IX, pp. 35-127, Academic Press, Nueva York.

2. Ko, R. (1983). Ultrasonic evaluation of interfacial properties in imperfect anisotropic layer-substrate. Tesis doctoral, The Ohio State University.

3. Nagy, P.B. y L. Adler,. (1988). Ultrasonic NDE of solid-state bonds: inertia and friction welds. J. of Nondestructive Evaluation, Vol. 7, Num. 3/4. 